\title{
Linguistic Production Abilities of 3-Year-Old Children Born Premature with Low Birth Weight
}

\author{
HOLLY K. CRAIG \\ JULIA L. EVANS \\ SAMUEL J. MEISELS \\ JAMES W. PLUNKETT \\ University of Michigan
}

Using norm-referenced and informal language sample analyses, this study describes the language production abilities of 30 children born premature with low birth weight. Only four of the subjects demonstrated clinically significant language problems at 3 years of age. The language problems of these four children did not appear to be related systematically to their birth weight, gestational age, length of neonatal hospitalization and severity of respiratory illness, socioeconomic status, family structure, or cognitive level and were best characterized by circumscribed expressive syntax diffi. culties.

Low birth weight and prematurity may put infants at risk for long-term developmental problems. Although these children appear more resilient than formerly supposed and though the incidence of major handicapping conditions has been decreasing (Vohr $\mathcal{E}$ Hack, 1982), more subtle cognitive and socioemotional problems continue to characterize this population (Meisels \& Plunkett, 1988; Plunkett \& Meisels, 1989; Plunkett, Meisels, Stiefel, Pasick, $\mathcal{E}$ Roloff, 1986). For example, more anxious-resistant patterns of mother-child attachment (Plunkett et al., 1986) and low performances across a variety of cognitive functions (Field, Dempsey, $\mathcal{E}$ Shuman, 1983) may characterize these children at later periods of development.

In the context of persistent cognitive and socioemotional sequelae to low birth weight and prematurity, it is not surprising that lan- guage problems have been observed among preterm infants. However, these observations are difficult to interpret for two major reasons. First, subject group heterogeneity is common within and across investigations that refer to low birth weight and prematurity. Ehrlich, Shapiro, Kimball, and Huttner (1973), for example, examined the communication skills of 81 5-year-old children with diverse high-risk neonatal histories. Many of the subjects were premature with low birth weight; however, other subjects demonstrated mild mental retardation, cerebral palsy, visual impairments, or significant sensorineural hearing loss. Although this type of subject sampling approach is important when gathering prevalence data for major handicapping conditions associated with high-risk populations, it obscures interpretation of the significance of the low language 
performances observed for a specific group. Further, language scores are reported typically as group data, masking individual performance. The difficulties involved when interpreting group data are compounded by the heterogeneity of most samples.

Second, most studies have included only formal language tests employing research designs that examine a broad range of developmental skills including language (Ehrlich et al., 1973; Rubin, Rosenblatt, \& Balow, 1973; Siegel, 1983; Siegel et al., 1982). Although potentially less efficient and more time consuming, language-sampling analysis techniques are more reliable, valid, and sensitive than formal tests for characterizing the language skills of young children (Lund E Duchan, 1988; Muma, 1978). Accordingly, the language measures employed in much of the research may have been both descrip. tively limited and insufficiently sensitive to reveal the nature of the language problems involved.

More recently a few studies have examined the language skills of children who were born premature with low birth weight more com. prehensively and systematically and have adopted language-sampling techniques. These studies examined language outcomes in terms of variables known to influence the developmental sequelae of low birth weight and prematurity, such as the presence and severity of respiratory illness, as well as factors known to have an effect on language development, such as hearing loss.

Hubatch, Johnson, Kistler, Burns, and Moneka (1985) examined the language abilities of infants born prematurely with a history of respiratory distress syndrome. They found that the children who were premature were significantly older when they attained the one-word stage than full-term control subjects. Some of the children born prematurely had conductive hearing losses, and the authors concluded that the language differ- ences observed between groups may have resulted from differing hearing status. The potentially important influences of race, gender, and socioeconomic status (SES) were not evaluated even though the subjects varied in these characteristics.

Kelsey and Barrie-Blackley (1976) found no significant language differences among children who were either preterm, full-term, and full-term but small-for-dates at approximately 4 years of age. The authors suggested that the relatively high socioeconomic status (SES) of their sample (all upper and middle class) may have excluded those children with low birth weights who were at risk for language delays.

A group of children born prematurely described by Field et al. (1983) demonstrated significantly shorter sentences, spoke less frequently in general, and demonstrated small. er productive vocabularies than full-term con. trols at 2, 3, and 4 years of age. Interestingly, these children also performed signficantly lower on the McCarthy Scales of Children's Abilities (McCarthy, 1972) for all subscales but memory. The Field et al. data are difficult to interpret, however. Are these differences reported for the preterm and full-term controls diagnostically significant? The absence of a norm-referenced language measure in this study yields inconclusive findings. These lower scores may be significant statistically but not clinically. Does the magnitude of the differences observed warrant enrollment in a formal language intervention program? In addition, the correspondingly lower performances on the McCarthy scales by the subjects born prematurely indicate that the children's language delays are associated with generalized cognitive delays. A broad flat profile of this type is suggestive. Perhaps the language differences experienced by infants with low birth weight who are born prematurely reflect less a specific linguistic impairment and more a generalized develop- 
mental lag. This issue relates to intervention implications as well.

Overall, the recent studies that focus more directly upon the language abilities of infants who were low birth weight and premature and use potentially more discriminating language-sampling analysis techniques reveal that a number of medical and demographic characteristics of the subjects influence their language development. Degree of respiratory illness, hearing loss, cognitive status, and socioeconomic status all seem to be poten. tially important factors for the language development of these children. The impact of gender remains unclear, though normally developing girls have a clear linguistic developmental advantage over boys (Cowen, Weber, Hoddenott, \& Klein, 1967; Halverson \& Waldrop, 1970; Koenigsknecht $\mathcal{E}$ Friedman, 1976; McCarthy, 1930; Ramer, 1976).

This investigation examined the language abilities of 3-year-olds who were born prematurely with low birth weight. At approximately 36 months, most children with normal language are at a critical period in their linguistic development: Simple sentence use has evolved, and complex syntax is emerging (Brown, 1973; Miller \& Chapman, 1981). The transition to complex syntax is typically an extremely difficult and protracted period for children with language problems (Leonard, 1972; Morehead $\mathcal{E}$ Ingram, 1973) and, therefore, represents a particularly revealing time for examining the linguistic skills of children who are born prematurely with low birth weight. In addition, the subjects were selected so that the influence of the following variables could be explored: neonatal risk level associated with degree of respiratory illness and length of hospitalization, birth weight, gestational age, SES, and gender. Language measures were derived from both norm-referenced and descriptive languagesampling procedures, examined both expressive and receptive modes, and explored lin- guistic production in terms of both syntax and vocabulary.

The following questions were posed:

1. At 3 years of age what is the linguistic status of children who were born premature?

2. How do their receptive and expressive skills compare?

3. Do these children have clinically significant language problems? If language problems are present, do children born prematurely differ from their peers in terms of risk level, birth weight, gestational age, socioeconomic status, gender, or family structure?

\section{METHOD}

\section{Subjects}

The subjects were 30 3-year-old children with a history of low birth weight and prematurity. All subjects were born weighing less than $2500 \mathrm{~g}$ (mean $=1660$; range $=850-2495$ ). All were gestationally younger than 38 weeks, with a mean gestational period for the group of 32 weeks and a range of 27 to 37 weeks. The average neonatal hospital stay for the group was 42 days, with a wide range of hospitalization ( 3 to 100 days).

At the time of this data collection, the children were approximately 3 years of age. The mean chronological age for the group was 38 months (range 36 to 40 months), with a corresponding corrected mean chronological age of 36 months (range 35 to 37.5 months). The corrected chronological ages reflect ad. justment for each child's short gestation. Adjusted chronological ages were used because the research literature on preterms is not yet clear on when to stop adjusting for prematurity; for the purposes of this investigation, the adjusted ages represented a more conserva. tive analytical approach.

All of the children were participating in a 
longitudinal investigation of the long-term effects of high-risk preterm birth. Children were identified from the case records of consecutive admissions to the neonatal intensive care unit of a large university hospital in the midwest. Families were recruited by letter and phone call. Of 82 families contacted, 65 agreed to participate, and 62 met the following subject exclusion criteria: absence of retrolental fibroplasia, multiple congenital anomalies, syndromes such as Down syndrome, microcephaly, Congenital Torch Infections, severe intrauterine growth retardation, or maternal drug addiction. These exclusion criteria were used to ensure that the infants enrolled in the study represented the population of infants with primary medical conditions associated with low birth weight and prematurity rather than some other diverse set of risk factors. One of the 62 potential subjects in this longitudinal study was excluded because of alterations in the investigation's protocol.

Each child in the longitudinal study was classified from perinatal and postnatal variables as high-, moderate, or low-risk. As determined by physical examinations and radiographic studies, children in the high-risk group had moderate to severe respiratory illness (no resolution of respiratory illness within 3 weeks of birth) and also had an initial hospitalization of more than 2 months. Children in the moderate-risk group had mild to moderate respiratory illness (resolution of respiratory illness within 3 weeks of birth) and hospitalizations initially between 1 and 2 months. Children in the low-risk group were free of respiratory illness and had been hospitalized for less than a month.

At 3 years, 55 of the 61 families $(85 \%)$ returned for follow-up. The questions posed in this language investigation required the ad. ditional exclusion of some children as potential subjects because of data collection problems, particularly failure to administer the
McCarthy scales (McCarthy, 1972) or sample collection with the father instead of the mother. Also, twins were excluded, as were nonwhite children and Hispanic and Oriental children.

Half of the subjects were males, half were females, and all of the subjects were Caucasian. Approximately two thirds of the subjects were from middle-class families; the others were from lower socioeconomic status families, based upon the Hollingshead Four. Factor Index of Socioeconomic Status (Hollingshead, 1975). Most of the children (73\%) were first born from families with both parents residing in the home $(90 \%$ were twoparent homes). Number of years of maternal education ranged from 11 to 18 , with a mean of 13.1 years. The subjects were selected to sample a full range of severity. Accordingly, $30 \%$ of the sample were high risk, $40 \%$ were moderate risk, and $30 \%$ were low risk.

As neonates, the Auditory Brain-Stem Evoked Responses (ABR) of the children were within normal limits, and none of the children had a history of significant hearing loss at age 3. An oral peripheral examination at the time of the study revealed unremarkable findings for all subjects. In addition, the children's performance on the McCarthy Scales of Children's Abilities (McCarthy, 1972) demonstrated that the general cognitive functioning of each child was within normal limits. Potential subjects were excluded if they failed to score above 85 on the General Cognitive Index based upon their corrected chronological ages. None of these chldren had participated in a formal early intervention program prior to this data collection at 3 years of age.

\section{Procedure}

Spontaneous language samples were collected from each child during mother-child interaction that was part of a larger data collection protocol including the administration 
of formal tests. During one part of the interaction, the mothers were instructed to encourage their children to play with the toys and to interact with them naturally as if they were playing together at home. This interaction involved play with a variety of ageappropriate toys, including cars and trucks, play telephones, building blocks, dolls, and puppets. A second component of the interaction involved the mothers in more structured play, including a lotto matching game, copying an Etch-A.Sketch pattern, and building a tower with large building blocks. The resulting 20-minute play interaction was successful in generating a rich corpus of child utterances across a range of free-play and semistructured tasks.

This procedure yielded a total language sample for analysis of 10 hours. The language samples were videotaped using a Beta videocassette recorder in a laboratory room arranged as a playroom.

The Preschool Language Scale (PLS) (Zimmerman, Steiner, $\mathcal{E}$ Pond, 1979) also was administered to each of these subjects. The PLS is composed of both auditory comprehension and verbal production tasks. Separate auditory comprehension and verbal ability quotients can be derived, and in this study they provided a basis for comparison of the children's expressive and receptive language skills.

\section{Scoring Procedure}

The language samples were transcribed orth. ographically and segmented into utterances. The first 50 consecutive child utterances composed of a subject-predicate relationship, the first 100 consecutive child utterances regardless of sentence form, and the first 50 utterances regardless of sentence structure formed the bases of the following three language-sampling scoring systems, respectively.

\section{Developmental Sentence Analysis}

(DSA) (Lee, 1974). This language-sampling procedure was designed to estimate the syntactic complexity of a child's utterances by evaluating production of eight major grammatical categories and overall grammatical correctness. For the purpose of this investigation, this standardized procedure served an "assessment" function, revealing whether the child's language development was within the statistically normal range. The analysis yields a composite Developmental Sentence Score (DSS); corresponding standardization data are available for children ages 2.0 to $6-6$ years. Performance below the 10th percentile for a child's chronological age was considered diagnostically significant.

2. Mean Length of Utterance (MLU) (criteria from Brown, 1973; Miller \& Chapman, 1981). Utterance length increases reliably with increasing structural maturity for normal language children, and MLU and Brown's corresponding Language Structural Stages provide a widely accepted way of indexing and comparing children's sentence growth. The MLU is computed by assigning each morpheme in a 100-utterance sample a value of 1 and dividing the sum of the morphemes by the 100 utterances in the sample. At 36 months, the predicted MLU is 3.16 , with a standard deviation of .694 (Miller $E$ Chap. man, 1981). Corresponding stage assignments are determined from the $M L U$ and the Upper Bound (the number of morphemes in the longest utterance of the sample). In this study, this language sample analysis was used to determine each child's language structural level. The language structural stage predicted for 36 months is Early IV (Miller \& Chapman, 1981), in which complex syntactic forms begin to emerge.

3. Type-Token Ratio (TTR) (Templin, 1957). The TTR is a long-standing and widely used measure of lexical diversity (Miller, 1981). It is computed by dividing the total number of words in a 50-utterance sample (tokens) by 
the number of those words that are different (types). This language sample analysis was used to assess each child's expressive vocab. ulary in this study. Type-token ratios should be 1:2 (approximately .5) for children between the ages of 3 and 8 years (Templin, 1957).

Although the stability and reliability of the MLU and TTR may be a concern for older, linguistically more mature children (Chabon, Kent-Udolf, $\mathcal{E}$ Egolf, 1982; Hess, Haug, $\mathcal{E}$ Landry, 1989), reliability is reportedly good for 3-year-olds (Brown, 1973; Templin, 1957). The MLUs and TTRs for each subject were scored using Lingquest 1 (Mordecai, Palin, $\mathcal{E}$ Palmer, 1985), a software program that computes these analyses automatically.

\section{Reliability}

A transcription reliability was established for each tape. An independent observer retranscribed a subset of the language samples collected for each child. These utterances were determined randomly, and the number transcribed corresponded to approximately $10 \%$ of each child's corpus and, therefore, $10 \%$ of the total sample of utterances. A comparison between the experimenter's and observer's transcribed words was $86 \%$ for morphemes. In addition, three children's transcripts were randomly selected and the DSS computed a second time by an independent observer. The percentile rank for each DSS was the same as that obtained by the original exam. iner, indicating good scoring reliability.

\section{RESCILTS}

The language skills of most of this sample of children born low birth weight and preterm appeared to be within normal expectations at approximately 3 years of age. Only four of the children demonstrated clinically significant language delays.

Craig, Evans, Meisels, E Plunkett
The DSS attained by most of the children ( $n=26 ; 83 \%$ of sample) was above the 25 th percentile for their corrected chronological ages on this measure of grammatical development (see Figure 1). Interestingly, the scores of all 26 children remained above the 10 th percentile when their uncorrected chronological ages were used as the basis for comparison. On the DSA the 4 remaining children (13\% of the sample) scored below the 10th percentile for their corrected chronological ages. The Developmental Sentence Scores of these children were significantly lower statistically (see Table 1) than the scores of the other children (independent $t=4.15$; $d f=2,28 ; p<.01)$. These 4 children are designated as subjects with language impairment in the subsequent discussions, and the other 26 children as subjects with normal language. The DSA profiles presented by these 4 children appeared generally consistent with each other. Overall, few higher-level

\section{FIGURE 1}

Cumulative Percentage Frequencies of Subjects for Each Developmental Sentence Score (DSS) Percentile Based Upon Corrected Chronological Ages

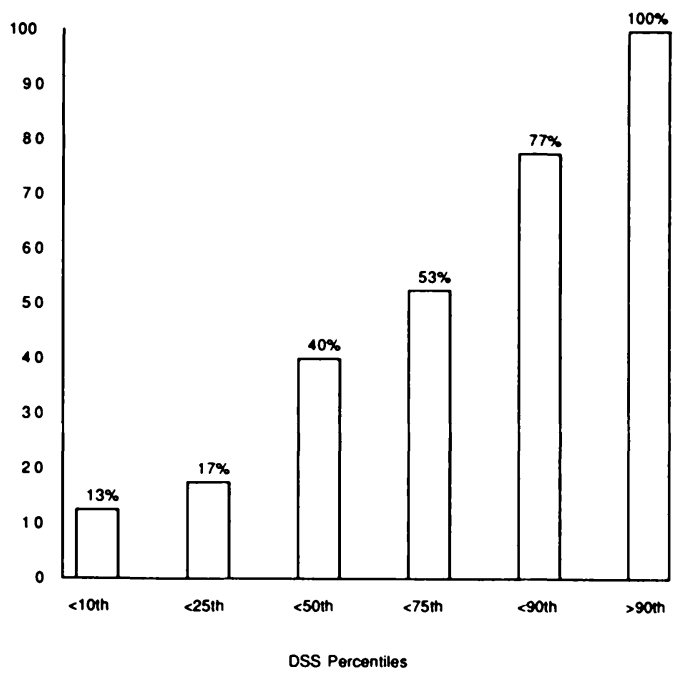


TABLE 1

Scores on the Language Measures and McCarthy Scales of Children's Abilities

\begin{tabular}{lcc}
\hline Measure & $\begin{array}{c}\text { Subjects with } \\
\text { Language } \\
\text { Impairments }(\boldsymbol{n}=\mathbf{4})\end{array}$ & $\begin{array}{c}\text { Subjects with } \\
\text { Normal } \\
\text { Language }(\boldsymbol{n}=\mathbf{2 6})\end{array}$ \\
\hline Developmental Sentence Scores* & & \\
M & 2.62 & 6.44 \\
SD & .47 & 1.81 \\
Mean Length of Utterance* & & 3.26 \\
M & 1.92 & .59 \\
SD & .60 & .48 \\
Type-Token Ratio & & .06 \\
$M$ & .54 & 129 \\
SD & .04 & 19 \\
Auditory Comprehension & 112 & 118 \\
Quotient & 19 & 15 \\
Verbal Ability* & 92 & 52 \\
Quotient & .17 & 8 \\
Perceptual & & 48 \\
$M$ & 50 & 8 \\
SD & 4 & \\
Motor & & \\
$M$ & 42 & \\
SD & 5 & \\
\hline
\end{tabular}

${ }^{*} p<.01$

structures were produced across all $8 \mathrm{gram}$. matical categories and, unlike the other 26 children, these 4 made only rare attempts at producing conjunctions.

The Mean Length of Utterance (MLU) values attained by these 4 children with language impairments were significantly different statistically from those of the 26 subjects with normal language (independent $t=4.23$; $d f=2,28 ; p<.01)$. The MLUs of 2 of these 4 children corresponded to Early Stage I, one to Stage II, and one to Stage III (Miller \& Chapman, 1981). Stages II and III reflected some subject overlap between those 4 children scoring below the 10 th percentile on the DSA and 6 of the 26 children scoring above the 25 th percentile on the DSA.

Stages I through III reflect the evolution of the simple sentence form (Brown, 1973). The most complex utterances produced by the four language-impaired children were all early developing syntactic sentence forms; for example; Subject \#1-“It go" and "Turn corner"; Subject \#2- "Why you coming in here?" and "How are the dog?"

Few of the children with normal language ( 6 children or $12 \%$ ) produced utterances characteristic of these early language developmental stages; in no instances were the children with normal language functioning as low as Stage I. Beyond Stage III, the auxiliary verb system is well-developed, and compound and complex sentence forms emerge (Brown, 1973). Some examples of the most complex utterances produced by this larger group of children with age-appropriate language skills follow: Subject \#7-“l'm pretending I'm a cow" and "I can driven them up 
like this"; Subject \#8-“"'Cause I don't know if her socks come off" and "Would you put her bottle in her mouth?"

The language samples collected from each child also were examined for the lexical diversity encoded by the children. The lexical Type-Token Ratios did not vary significantly (see Table 1) for the four language-impaired children (independent $t=1.96 ; d f=2,28 ; p$ $>$.05). This finding indicates that the language production differences noted for these four children were syntactically rather than lexically based.

On the Preschool Language Scale (Zimmerman, 1979), the Auditory Comprehension Quotients of the four subjects with language impairments were lower than those of the children with age-appropriate language skills but this difference was not significant statistically (independent $t=1.62 ; d f=2,28 ; p$ > .05). However, the Verbal Ability Quotients of the two groups did differ signficantly (independent $t=3.22 ; d f=2,28 ; p<.01)$. These findings indicate that the expressive differences observed for these four children were not associated with parallel receptive language differences.

The language differences observed for these four subjects with language impair- ments seemed unrelated to factors typically considered important to the long-term consequences of low birth weight and prematurity. The presence of clinically significant language delays did not vary systematically with neonatal birth weight, gestational age, length of hospitalization, maternal education, or risk group (see Table 2). Further, the child with the lowest birth weight $(850 \mathrm{~g})$ scored above the 90th percentile on the DSA, whereas, as can be seen in Table 2, one of the children with language impairments had a relatively high birth weight $(2211 \mathrm{~g})$. The longest periods of neonatal hospitalization (100 and 94 days) were by children who scored above the 25th percentile and above the 90th percentile, respectively, on this same measure. Subject $\# 4$, in contrast, had a very short hospital stay of 5 days. It is interesting that none of the four subjects had been designated as high risk at birth based upon respiratory illness and hospitalization.

Similarly, the family characteristics of the four subjects with language impairments did not vary systematically with their language problems. Half of these children were from lower and half from middle socioeconomic status homes. Birth order of the subject appeared unrelated also, as half of the children

\section{TABLE 2}

\section{Characteristics of the Children with Language Impairments}

\begin{tabular}{lcccc}
\hline & \multicolumn{4}{c}{ Subject } \\
Characteristic & $\mathbf{1}$ & $\mathbf{2}$ & $\mathbf{3}$ & $\mathbf{4}$ \\
\hline Birth weight (grams) & 1247 & 1920 & 1900 & 2211 \\
Gestational period (weeks) & 32 & 32 & 36 & 36 \\
Hospitalization (days) & 43 & 16 & 39 & 5 \\
Corrected age (months) & 34.5 & 35 & 36.5 & 35 \\
Uncorrected age (months) & 37 & 37 & 37.5 & 36 \\
Risk group & Mod. & Low & Mod. & Low \\
Gender & Male & Male & Male & Male \\
Socioeconomic status & Middle & Middle & Low & Low \\
Parity & First & First & Later & Later \\
Number of parents in home & 2 & 2 & 2 & 2 \\
Maternal education (years) & 13 & 12 & 11 & 12 \\
\hline
\end{tabular}

Craig, Evans, Meisels, E Plunkett 
were first-born and half were later-born. The children with language impairments all came from two-parent families, indicating that family structures were not contributing factors; the number of years of maternal education was comparable also (language-impaired mean $=12.0$ years; remaining sample mean $=12.7$ years).

Another subject characteristic consistent across all the subjects with language impairments, in addition to the absence of high-risk birth histories and the presence of both parents in the home, was the child's gender. All four of these children were boys (see Table 2).

On the McCarthy Scales of Children's Abilities (McCarthy, 1972), the group of four subjects with language impairments were not different statistically from the other subjects on either the perceptual (independent $t=$ 0.63 ; $d f=2,28 ; p>.05$ ) or the motor (independent $t=1.43 ; d f=2,28 ; p>$.05) scales (corrected for prematurity). These scales were selected to permit comparisons between groups in terms of nonverbal skills. This finding indicates that the decreased language skills of these four children were not associated with some generalized cognitive delay.

\section{DISCUSSION}

Considerable literature to date contrasts sharply with the findings of this study. Language problems within the low birth weight preterm population have been reported for more than 20 years (for example, DeHirsch, Jansky, \& Langford, 1966). Differences between subjects in the present study and those reported previously may account for these discrepant findings. The present study also involved a level of subject sample control not available to date for language research with this population.

The language measures obtained were treated both as group data and as individual data. In addition, the stratification of the subject sample on variables known to be important to the understanding of language development, as well as to those factors influencing low birth weight and prematurity, permitted the interpretation of individual differences in language in a manner not possible within previous investigations.

Overall the findings of this investigation support an emerging consensus in the literature that the child born low birth weight and preterm is not as fragile as once supposed. Normal language development may be a likely outcome for many of these children. This study indicates that this prognosis extends to language development as well as to some other behavioral domains.

Within this context, it seems imperative to identify subgroups of infants born premature with low birth weight who are at risk for specific long-term difficulties. This study contributes to this endeavor by revealing that infants born premature with low birth weight who are free of other major handicapping conditions ultimately are no more at risk for language disorder than the general population. Although incidence data for developmental language problems are difficult to obtain, the $13 \%$ identified within this sample closely approximates the rates (approximately $12 \%$ ) estimated most recently for the general population (Beitchman, Nair, Clegg, \& Patel, 1986). These findings highlight the need for carefully controlled subject samples, as proposed previously by other language researchers (Kelsey \& Barrie-Blackley, 1976; Siegel et al., 1982). This type of approach to subject sampling will permit definition of subgroups at risk for particular disorders and reduce the need for broad-band, essentially undifferentiated approaches to assessment and clinical intervention with infants who are at high risk.

Four of the 30 children in this sample demonstrated clinically significant language delays on the DSA. Studies have examined 
the reliability and validity of the DSA and report that this instrument is both reliable and valid (Koenigsknecht, 1974). To the extent that the DSA is an accurate index of language problems, only 4 children in this investigation appeared to have significant language impairments. The close correspondence of 2 of the subjects with language impairments to the stages of 6 children with normal language may be a positive indicator for language potential. Unfortunately, longterm follow-up of these subjects was not possible.

The four children with language impairments presented relatively circumscribed linguistic difficulties that were expressive and syntactic in nature. Hubatch et al. (1985) reported poor receptive as well as expressive language performances by their subjects who were high risk; however, half of their subjects had hearing losses that may have related to their poor auditory comprehension skills. Siegel (1983) found no differences between subjects who were full-term and preterm at age 5 years on a test of single-word receptive vocabulary. This report provides some support for the present findings; together they indicate that when language problems do appear solely as a consequence of low birth weight and prematurity, they may be primarily expressive syntax problems.

Overall, the linguistic expression of these four children was problematic though auditory comprehension was not. This expressive language problem appears specifically linguistic. It is not associated with a generalized cognitive delay or significant neurological or sensory defects. The birth weight, gestational age, length of neonatal hospitalization and severity of respiratory illness, SES, birth order, maternal education, and presence of two parents in the home did not distinguish these children with language impairments from their peers. The linguistic profile presented by these four children is consistent with the expressive subgroup of the population of children with language disorders designated as "specifically language impaired" (Stark E Tallal, 1981; Wolfus, Moscovitch, E Kinsbourne, 1980). Their linguistic difficulties occur in the absence of clinically signfiicant neurological impairment, hearing loss, emotional problems, or sensory-motor defects, and the children's general intelligence appears to be within the normal range.

The absence of a clear relationship between environmental factors and clinical language problems is suggestive. This investigation had a relatively small sample size, however. Confirmation of these findings is needed with additional research that also controls for potentially confounding influences or ambiguous relationships.

All four children demonstrating poor expressive language skills were boys. By the age of 3, developmental language problems are more prevalent among boys than girls by a ratio of approximately 2 to 1 (Silva, 1980; Stevenson \& Richman, 1976). Unfortunately, little is known about the nature of the differences observed for boys and girls with language disorders. This issue warrants further investigation.

In conclusion, this study reveals that the language development of most infants born premature, with low birth weight, and at minimal risk based upon biological and environmental factors, is within normal expectations by 3 years of age. Language developmental outcomes appear quite good for children whose neonatal difficulties include low birth weight, prematurity, and various levels of respiratory illness but who do not have other major handicapping conditions. Those children who demonstrate language difficulties at 3 years seem best characterized by circum. scribed linguistic production problems similar to children with language disorders diag. nosed as specifically language impaired. 


\section{REFERENCES}

Beitchman, J., Nair, R., Clegg, M., \& Patel, P. (1986). Prevalence of speech and language disorders in 5-year-old kindergarten children in the Ottawa-Carleton region. Journal of Speech and Hearing Disorders, 51, 98-110.

Brown, R. (1973). A first language: The early stages. Cambridge: Harvard University Press.

Chabon, S., Kent.Udolf, L., \& Egolf, D. (1982). The temporal reliability of Brown's Mean Length of Utterance (MLU $M$ M) Measure with PostStage V Children. Journal of Speech and Hear. ing Research, 25, 117-124.

Cowen, P., Weber, J., Hoddenott, B., \& Klein, J. (1967). Mean length of spoken response as a function of stimulus, experimenter, and sub. ject. Child Development, 38, 191-203.

DeHirsch, K., Jansky, J.J., \& Langford, W.S. (1966). Comparisons between prematurely born and maturely born children at three age levels. American Journal of Orthopsychiatry, 36, 616.628.

Ehrlich, C.H., Shapiro, E., Kimball, B.D., \& Huttner, M. (1973). Communication skills in five-yearold children with high risk neonatal histories. Journal of Speech and Hearing Research, 16, 622.629.

Field, T., Dempsey, J., \& Shuman, H. (1983). Fiveyear follow-up of preterm respiratory distress syndrome and post-term post-maturity syndrome infants. In T. Field \& A. Sostek (Eds.), Infants bom at risk: Physiological, perceptual, and cognitive processes (pp. 317.335). New York: Grune $\mathcal{E}$ Stratton.

Halverson, C., \& Waldrop, M. (1970). Maternal behavior toward own and other preschool children: The problem of "ownness." Child Development, 41, 839-845.

Hess, C., Haug, H., \& Landry, R. (1989). The reliability of Type-Token Ratios for the oral language of school age children. Journal of Speech and Hearing Research, 32, 536-540.

Hollingshead, A.B. (1975). Four factor index of social status. Unpublished manuscript. New Haven, CT: Yale University.

Hubatch, L., Johnson, C., Kistler, D., Burns, W., \& Moneka, W. (1985). Early language abilities of high-risk infants. Journal of Speech and Hear. ing Disorders, 50, 195-207.
Kelsey, S., \& Barrie-Blackley, S. (1976). The language of low-birthweight children at four years: Preliminary report. Developmental Med. icine and Child Neurology, 18, 753.758.

Koenigsknecht, R. (1974). Statistical information on Developmental Sentence Analysis. In L. Lee, Developmental sentence analysis (pp. 222-268). Evanston, IL: Northwestern University Press.

Koenigsknecht, T., \& Friedman, P. (1976). Syntax development in boys and girls. Child Develop. ment, 47, 1109-1115.

Lee, L. (1974). Developmental Sentence Analysis. Evanston, IL: Northwestern University Press.

Leonard, L. (1972). What is deviant language? Journal of Speech and Hearing Disorders, 37, 427.447.

Lund, N.J., \& Duchan, J.F. (1988). Assessing children's language in naturalistic contexts. Englewood Cliffs, NJ: Prentice-Hall.

McCarthy, D. (1930). The language development of the preschool child. Cited in Y. Brackbill (Ed.), Infancy and early childhood (1967). New York: The Free Press.

McCarthy, D. (1972). McCarthy Scales of Children's Abilities. San Antonio, TX: Psychological Corp.

Meisels, S.J., E Plunkett, J.W. (1988). Developmental consequences of preterm birth: Are there long-term effects? In P.B. Baltes, D.L. Featherman, \& R.M. Lerner (Eds.), Life-span develop. ment and behavior (Vol. 9; pp. 87-128). Hillsdale, NJ: Lawrence Erlbaum.

Miller, J. (1981). Assessing language production in children: Experimental procedures. Baltimore: University Park Press.

Miller, J., \& Chapman, R. (1981). The relation between age and mean length of utterance in morphemes. Journal of Speech and Hearing Research, 24, 154-161.

Mordecai, D., Palin, M., \& Palmer, C. (1985). Ling. quest 1: Language sample analysis. San Antonio, TX: Psychological Corp

Morehead, D., \& Ingram, D. (1973). The development of base syntax in normal and linguistically deviant children. Journal of Speech and Hearing Research, 16, 330-352.

Muma, J. (1978). Language handbook: Concepts, assessment, intervention. Englewood Cliffs, NJ: Prentice-Hall. 
Plunkett, J., \& Meisels, S. (1989). Socio-emotional adaptation of preterm infants at three years. Journal of Infant Mental Health, 10, 117-131.

Plunkett, J.W., Meisels, S.J., Stiefel, G.S., Pasick, P.L., $\mathcal{E}$ Roloff, D.W. (1986). Patterns of attachment among preterm infants of varying biological risk. Journal of the American Academy of Child Psychiatry, 25, 794-800.

Ramer, A. (1976). Syntactic styles in emerging language. Journal of Child Language, 3, 49-62.

Rubin, R., Rosenblatt, C., \& Balow, B. (1973). Psychological and educational sequelae of prematurity. Pediatrics, 52, 352.363.

Siegel, L. (1983). The prediction of possible learning disabilities in preterm and full-term children. In T. Field \& A. Sostek (Eds.), Infants bom at risk: Physiological, perceptual, and cog. nitive processes (pp. 295-315). New York: Grune \& Stratton.

Siegel, LS., Saigal, S., Rosenbaum, P., Morton, R.A., Young, A., Berenbaum, S., \& Stoskopf, B. (1982). Predictors of development in preterm and full-term infants: A model for detecting the at risk. Joumal of Pediatric Psychology, 7, 135-148.

Silva, P. (1980). The prevalence, stability and significance of developmental language delay in preschool children. Developmental Medicine and Child Neurology, 22, 768.777.
Stark, R., \& Tallal, P. (1981). Selection of children with specific language deficits. Journal of Speech and Hearing Disorders, 46, 114-122.

Stevenson, J., \& Richman, N. (1976). The preva. lence of language delay in a population of three-year-old children and its association with general retardation. Developmental Med. icine and Child Neurology, 18, 431.441.

Templin, M. (1957). Certain language skills in children. Minneapolis: University of Minnesota Press.

Vohr, B.R., \& Hack, M. (1982). Developmental follow-up of low birthweight infants. Pediatric Clinics of North America, 29, 1441-1454.

Wolfus, B., Moscovitch, M., $\mathcal{E}$ Kinsbourne, M. (1980). Subgroups of developmental language impairment. Brain and Language, 9, 152-171.

Zimmerman, I.L., Steiner, V.G., \& Pond, R. (1979). Preschool Language Scale. San Antonio, TX: Psychological Corp.

We would like to thank our colleague, Dietrich Roloff, for his overall assistance in this study, and we extend our gratitude to the parents and children who participated in it. This work was supported, in part, by a grant from the March of Dimes Birth Defects Foundation.

Address all correspondence to Holly K. Craig, PhD, Communicative Disorders Clinic, The University of Michigan, 1111 E. Catherine Street, Ann Arbor, MI 48109.2054. 